\title{
Dual Polarization THz Time Domain Spectroscopy System
}

\author{
Yang $\mathrm{Yu}^{1), 2)}$, Guozhong Zhao ${ }^{1), 2), *}$, Giorgio Savini ${ }^{2), 3), *}$ \\ 1) Department of Physics , Capital Normal University, ${ }^{2)}$ Key Lab of THz Optoelectronics, Ministry of Education, Beijing, China. ${ }^{3)}$ Optical \\ Science Laboratory, Physics and Astronomy Department, University College London, London, UK \\ *Corresponding author, Email: guozhong-zhao@cnu.edu.cn, g.savini@ucl.ac.uk
}

\begin{abstract}
In many THz Time Domain Spectroscopy configurations, the separate acquisition of background and sample measurements have significant impact on data quality due to the need to perform subsequent measurements as well as the change in beam coupling geometry due to the removal of the sample in the reference measurement. In this short paper, we report on the design and build of a dual-polarization TDS system to perform synchronous background/sample measurements. This is achieved through the splitting of the PCA-generated THz beam with a $\mathrm{THz}$ polarizer to obtain two orthogonal polarized beams which are balanced and modulate the indices of two separate crystals on which the probe beam (also split) is reflected off before being detected. The two branches of the system show good similarity, and can be used to perform background and sample measurements synchronously, thus not being affected by changes in temperature and humidity, as well as small fluctuations in source power. Small differences in detector optical efficiency can be calibrated out at the start of a measurement session.
\end{abstract}

\section{Index Terms-Spectroscopy; TDS; polarization; polarimetry}

\section{INTRODUCTION}

With the rapid development of terahertz radiation generation and detection technology, terahertz time-domain spectroscopy(THz-TDS) technique has steadily and gradually matured over the last 30 years [1]. For example, terahertz emitters [2-4] and detectors [5-6] have improved significantly as a result of recent advances in semiconductor fabrication. Until recently, polarized-dependent terahertz spectroscopy has attracted the interest of researchers due to the importance of the anisotropic dielectric properties of materials in chemical, physical and biochemical fields. Such as, anisotropy of metamaterials, biomolecules and inorganic crystals at terahertz frequencies. Yet in recent years, several groups have designed and fabricated polarization sensitive detectors and emitters that are capable of measuring the full transverse timedependent terahertz waveform. For example, a novel threecontact photoconductive detector [7-11] was developed to simplify the direct detection of polarized signals. This was then replicated and improved in many other works [12-18] all compared in Fig. 1. These works make it possible to measure the polarization properties of the material.

In this paper, we show how a terahertz polarizer combined with the duplication of the detection chain of a standard TDS system allows to achieve a polarization sensitive terahertz time-domain spectroscopy system to achieve the study of the polarization properties of the material. Compared with other systems that measure polarization-related properties, the two branches of this system show good similarity and can simultaneously detect the sample and reference signal, avoiding the large uncertainties introduced by the variation of temperature, humidity, laser source power and other temporal variations.

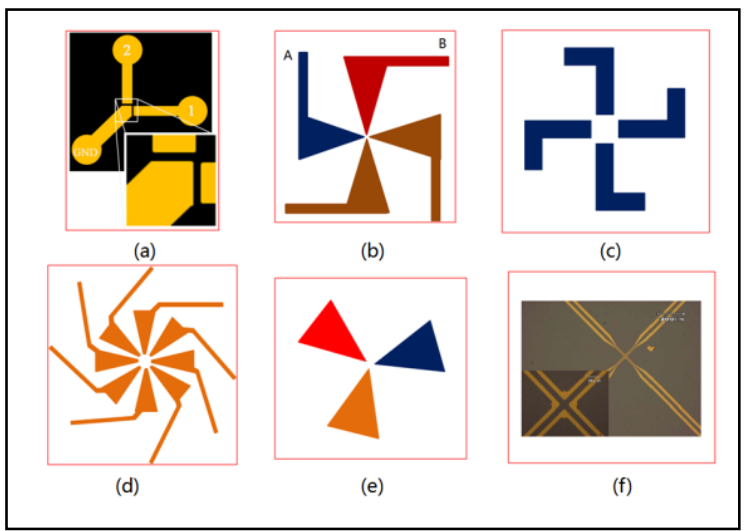

Fig. 1. Schematic diagram of polarization - sensitive terahertz photoconductive antenna.

\section{EXPERIMENT METHOD}

\section{A. Experimental device}

Based on the low-temperature grown gallium arsenide photoconductive antenna, a set of dual polarization $\mathrm{THz}$ time domain spectroscopy system is proposed. The system mainly includes: a mode-locked Ti:sapphire oscillators supplying 100 fs pulses with a repetition rate of $82 \mathrm{MHz}$, average power $3400 \mathrm{~mW}$ and the center wavelength of $800 \mathrm{~nm}$; a lowtemperature grown gallium arsenide photoconductive antenna emitter triggered by femtosecond laser radiating terahertz power; optical path delay stage; THz polarizer [19]; and two terahertz detection systems (ZnTe crystal and lock-in amplifier and so on ).

The optical polarization beam splitter (PBS) divides the femtosecond laser into two beams. The first beam referred to as the probe beam (reflected by the polarization beam splitter) is further divided into two beams by another beam splitter. Then each beam passes through a half-wave plate and a polarizer to adjust the polarization of the probe beams, finally each beam is focused with a lens to a $1 \mathrm{~mm}$ thick $\mathrm{ZnTe}$ crystal. The second beam is the pump beam(transmitted through the beam splitter), and generates a terahertz wave by exciting the photoconductive antenna. The forward propagating $\mathrm{THz}$ beam is collimated off a gold-plated off-axis paraboloid mirror(M1), and then divided into two beams by the terahertz polarizer positioned at 45 degrees with respect to the incident beam. For reference, the beam propagating through the terahertz polarizer is named "pump beam 1"and the reflected beam from the terahertz polarizer is named "pump beam 2". The terahertz polarizer is adjusted so that the polarization state of the pump beam 1 is rotated by 45 degrees along the clockwise 
direction and the polarization state of the pump beam 2 similarly along the counterclockwise direction. Each pump beam is then focused on a probe crystal $\mathrm{ZnTe}$ at the position of reflection of the relevant probe beams, modulated by the $\mathrm{THz}$ singnal and finally detected by two differential detectors (Detector 1 and Detector 2), respectively. The differential output signals are ampified by a lock-in amplifier and sent to a computer for processing.

At the beginning of the measurement session, small differences in detector optical efficiency and responsivity can be calibrated so that the two reference signals are in good agreement, The sample can then be placed in the pump beam 1 and measurements taken simultaneously of the sample (beam 1) and its reference (beam 2). The sample can then be rotated 90 degrees for it to be measured at the orthogonal polarization. Therefore, this system can synchronize the measurement of reference and sample data, avoiding the effects of many interfering factors.

\section{B. Data analysis}

The following is a short recall of the refractive index and birefringence theory employed subsequently in our analysis. If the time delay of the pulse propagation in the crystal is much longer than the duration of the pulse, we can ignore the terahertz pulse and sample internal reflection of the terahertz pulse overlap, during the sample measurement process. In this case, the Fabry-Perot interference term can be ignored, and the real part of the refractive index can be written as

$$
\mathrm{n}^{[\mathrm{u}]}(\omega)=1+\frac{c}{\omega d}\left(\phi_{\text {sam }}^{[u]}(\omega)-\phi_{r e f}^{[u]}(\omega)\right)
$$

The imaginary part of the refractive index can be written as

$$
\kappa^{[u]}(\omega)=\frac{c}{2 \omega d}\left[\frac{\varepsilon_{\text {sam }}^{[u]}(\omega) / \varepsilon_{r e f}^{[u]}(\omega)}{t_{12}^{[u]}(\omega) t_{21}^{[u]}(\omega)}\right]
$$

$\varepsilon^{[u]}$ and $\phi^{[u]}$ are the amplitude and phase after Fourier transform, respectively, $\mathrm{d}$ is the thickness of the sample, $t_{12}^{[u]}(\omega)=2 /\left(n^{[u]}(\omega)+1\right)$ and $t_{21}^{[u]}(\omega)=2 n^{[u]}(\omega) /\left(n^{[u]}(\omega)+1\right)$ are the Fresnel transmission coefficients at the surface of the sample.

Birefringence is defined as $\delta n(\omega)=n^{[E]}(\omega)-n^{[O]}(\omega)$, therefore

$$
\delta n(\omega)=\frac{c}{\omega d}\left[\phi_{\text {sam }}^{[E]}(\omega)-\phi_{\text {ref }}^{[E]}(\omega)-\phi_{\text {sam }}^{[O]}(\omega)+\phi_{r e f}^{[O]}(\omega)\right]
$$

\section{MEASUREMENT RESULTS AND DISCUSSION}

With the system at room temperature $\left(20^{\circ} \mathrm{C}\right.$ and humidity $48.3 \%$ ), and no sample present, the time domain signal ratio of the pump beam1(called $\mathrm{T}$ in Fig. 3)to the pump2(called $\mathrm{R}$ in Fig. 3) is shown in Fig. 3.
It is evident that the camparison of the signal from the two branches of the spectrometer are consistent. We use the fast Fourier transform of the data measured by the system and the equation(4) to calculate the sapphire birefringence, as shown in Fig. 4.

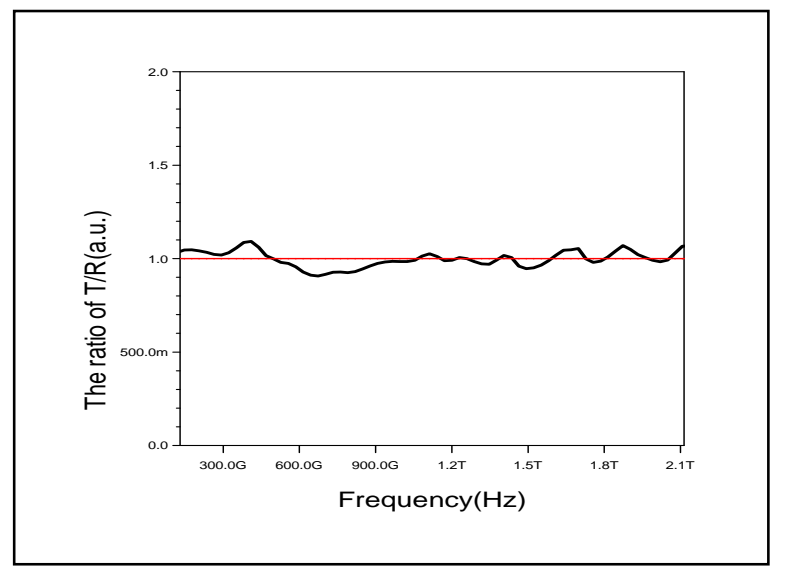

Fig. 3. the time domain signal ratio of the pump beam1(T)to the pump2(R).

In Fig. 4, at a similar environment conditions, a plot of two refraction indices of sapphire are shown. At $1.5 \mathrm{THz}$, the extraordinary refractive index is 3.424 , and the ordinary refractive index is 3.074. These values are very similar to those of Cook [20], Loewenstein [21], Russell [22] at room temperature and $1.5 \mathrm{THz}$, with a refractive indices respectively of 3.428 and 3.076 .

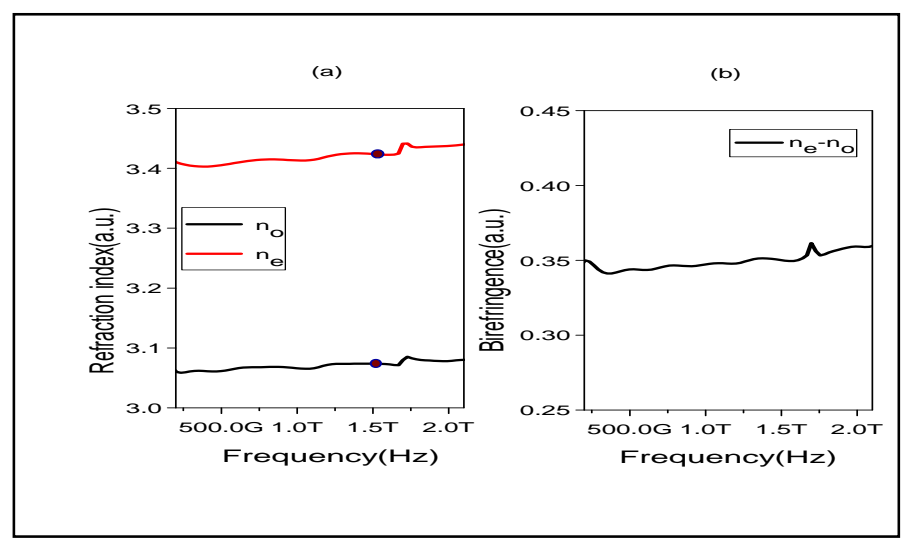

Fig. 4. (a) Ordinary and extraordinary refractive indices of sapphires measured by dual polarization THz-TDS, (b) Birefringence of sapphire calculated from (a).

\section{CONCLUSION}

In the experiment, the system of signal to noise ratio of 350 : 1 or more, the system is stable and easily reproduced. In summary, a dual polarization terahertz time-domain spectroscopy system is designed. The measurement data of sapphire crystals is in good agreement with the value measured by Loewenstein et al., the measurement of birefringence properties of sapphire crystals proved the effectiveness of the system. This system is useful for further study of optical activity and circular dichroism and other properties of polarized material. In addition, our finding in this 
study can lead to further study of dual polarization synchronous emission systems.

\section{REFERENCES}

[1] G.ZH. Zhao, "Terahertz Time-Domain Spectroscopy and Its Applications,” Journal of Electronic Science and Technology12(2), 146149 (2014)

[2] Y. C. Shen, P. C. Upadhya, E. H. Linfield, and H. E. BeereA. G. Davies, "Ultrabroadband terahertz radiation from low-temperature-grown GaAs photoconductive emitters,” Appl. Phys. Lett. 83(15) 3117-3119 (2003).

[3] E. Castro-Camus, J. Lloyd-Hughes, and M. B. Johnston, "Threedimensional carrier-dynamics simulation of terahertz emission from photoconductive switches," Phys. Rev. B, 71, 195301 (2005).

[4] Huang Zhen. Yu Bin2. Zhao Guozhong, "The THz Emission Properties of Small-Aperture Photoconductive Antenna," Chinese Journal of Lasers. 37(1), 110-114 (2010).

[5] E. Castro-Camus, L. Fu, J. Lloyd-Hughes, H. H. Tan, C. Jagadish, and M. B. Johnston, "Photoconductive response correction for detectors of terahertz radiation,” J. Appl. Phys. 104, 053113 (2008).

[6] M.Tani, K.Sakai, H.Mimura, "Ultrafast photoconductive detectors based on semi-insulating GaAs,” J.Appl. Phys.36 (9), 1175-1178(1997).

[7] E. Castro-Camus, J. Lloyd-Hughes, M. B. Johnston, M. D. Fraser, H. H Tan, and C. Jagadish, "Polarization-sensitive terahertz detection by multicontact photoconductive receivers," Appl. Phys. Lett. 86, 254102 (2005).

[8] E. Castro-Camus, "Polarization-resolved terahertz time-domain spectroscopy,” J. Infrared Milli Terahz Waves. 33(4), 418-430(2012).

[9] E. Castro-Camus and M. B. Johnston, "Extraction "of the anisotropic dielectric properties of materials from polarization-resolved terahertz time-domain spectra,” J. Opt. A, Pure Appl. Opt. 11(10), 105206 (2009).

[10] E. Castro-Camus and M. Alfaro, "Photoconductive devices for terahertz pulsed spectroscopy: A review," Photonics Res. 4(3), A36-A42 (2016).

[11] E. Castro-Camus, J. Lloyd-Hughes, M. D. Fraser, H, H, Tan, C, Jagadish and M. B. Johnston, "Detecting the full polarization state of terahertz transients", Proc. SPIE 6120, 61200Q, (2005).
[12] G. Niehues, S. Funkner, D. S. Bulgarevich, S. Tsuzuki, T. Furuya, K. Yamamoto, M. Shiwa, and M. Tani, "A matter of symmetry: terahertz polarization detection properties of a multi-contact photoconductive antenna evaluated by a response matrix analysis," Opt. Express 23(12), 16184-16195 (2015).

[13] D. S. Bulgarevich, M. Watanabe, M. Shiwa, G. Niehues, S. Nishizawa, and M. Tani, "A polarization-sensitive 4-contact detector for terahertz time-domain spectroscopy," Opt. Express 22(9), 10332-10340 (2014).

[14] A Hussain and S. R. Andrews, "Ultrabroadband polarization analysis of terahertz pulses," Opt. Express 16(10),7251-7257 (2008).

[15] H. Suo, K. Takano, S. Ohno, H. Kurosawa, K. Nakayama, T. Ishihara, and M. Hangyo, "Polarization property of terahertz wave emission from gammadion-type photoconductive antennas," Appl. Phys. Lett. 103(11), 111106 (2013).

[16] D. S. Bulgarevich, M. Watanabe, M. Shiwa, G. Niehues, H. Kitahara, and M. Tani, "Polarization-variable emitter for terahertz time-domain spectroscop," Optics Express. 24(24), 27160-27165 (2016).

[17] H. Makabe, Y. Hirota, M. Tani, and M. Hangyo, "Polarization state measurement of terahertz electromagnetic radiation by three-contact photoconductive antenna," Opt. Express 15(18), 11650-11657 (2007).

[18] Y. Hirota, R. Hattori, M. Tani, and M. Hangyo, "Polarization modulation of terahertz electromagnetic radiation by four-contact photoconductive antenna," Opt. Express 14(10), 4486-4493 (2006).

[19] A. Mitsuishi, Y. Yamada, S. Fujita, and H. Yoshinaga, "Polarizer for the far-infrared region," Journal of the Optical Society of America. 50(5), 433-436 (1960)

[20] W.B. Cook and S. Perkowitz, "Temperature dependence of the farinfrared ordinary-ray optical constants of sapphire," Appl. Opt. 24(12), 1773-1775 (1985).

[21] E.V. Loewenstein, D.R. Smith, R.L.Morgan, "Optical constants of far infrared materials. 2: crystalline solids," Appl. Opt.12(2), 398-406, (1973).

[22] E. E. Russell and E. E. Bell, "Optical constants of sapphire in the far infrared," J. Opt. Soc. Am. 57, 543-544 (1967). 